\title{
Presentation and outcome of tuberculous meningitis among children: experiences from a tertiary children's hospital
}

\author{
Nabukeera- Barungi Nicolette, ${ }^{1}$ Wilmshurst Jo, ${ }^{2}$ Rudzani Muloiwa, ${ }^{2}$ Nuttall James ${ }^{2}$
}

1. Makerere University College of Health Sciences, Kampala, Uganda.

2. Red Cross War Memorial Children's Hospital and the School of Child and Adolescent Health, University of Cape Town.

\begin{abstract}
Background: Diagnosis of tuberculous meningitis (TBM) is complicated and outcome is poor especially in resource limited settings. Early diagnosis and prompt treatment are vital in effective treatment. We set out to describe experiences in the management and immediate outcome of TBM a tertiary-level children's hospital in a high HIV and tuberculosis co-infection setting.

Methods:This retrospective study included children who were diagnosed with TBM in the year 2009. A pre-coded questionnaire was used to extract data on presentation, diagnostics, treatment and outcome at the time of hospital discharge. Data was analyzed using STATA statistical package (StataCorp, Version 11).

Results: Of the 40 children diagnosed with TBM, 6 (15\%) had definitive TBM, 17 (42.5\%) had probable TBM and 17 (42.5\%) had possible TBM. The cerebrospinal fluid (CSF) chemistry and cells were abnormal in 39/40 (98\%). Mantoux test was reactive in 16/29 (55\%) and 17/30 (57\%) had Chest X-rays suggestive of tuberculosis. Only $3 / 21$ (14\%) had positive sputum tuberculosis culture and $89 \%(32 / 36)$ had neuro-imaging abnormalities. Outcome at discharge was; $8 \%$ died, $49 \%$ improved with neurological sequelae and $43 \%$ improved without sequelae. Having TBM stage 3 at admission was associated with mortality $(\mathrm{p}=0.001)$.

Conclusions: Most children had early diagnosis of TBM and mortality was lower than in previous studies. We recommend a larger prospective study to further understand the outcome of TBM.

Key words:Tuberculous meningitis, children, presentation, outcome, Africa

African Health Sciences 2014;14(1): 143-149 http://dx.doi.org/10.4314/ahs.v14i1.22
\end{abstract}

\section{Introduction}

Tuberculosis (TB) is a major public-health problem with around 9 million new cases and 2 million deaths estimated to occur each year. ${ }^{1}$ About one-third of the world's population has latent TB with a lifetime risk of TB disease of $10 \% .^{2}$ However, among those coinfected with HIV and latent TB infection, the risk for TB reactivation is $10 \%$ per year. ${ }^{3}$ About $20 \%$ of TB is extra-pulmonary disease. ${ }^{4}$ Tuberculous meningitis is the most severe form of extra pulmonary tuberculosis. Its exact incidence and prevalence are not known in resource poor countries but in developed countries it ranges between 0 and 1 per 100,000 population. ${ }^{5}$ In South Africa, the incidence of TBM in Western Cape

\section{Correspondence author:}

Nicolette Nabukeera-Barungi

Department of Paediatrics and Child Health

School of Medicine

Makerere University College of Health Sciences

Tel: +256 772435166

Email: nicolettebarungi@gmail.com province was 24 per 100,000 population among children aged 0 to 4 years for the period of 1985-1987. ${ }^{6}$ The high prevalence of HIV and TB in this region led to a rise in TBM. ${ }^{7,8}$ In the Western Cape province, TBM was the leading cause of meningitis among children below 13 years of age. ${ }^{7}$

Diagnosis of TBM is complicated because of its non specific clinical presentation which may be acute, sub acute or chronic. It may be febrile or afebrile. The signs and symptoms of stage $1 \mathrm{TBM}$ disease are non specific and relate more to primary lung infection than neurological disease. ${ }^{5}$ TBM mainly affects young children with the mean age ranging between 23 and 49 months. ${ }^{9,10,11}$ Diagnosis requires a high index of suspicion from the clinician. The Mantoux test is positive in only 30 to $50 \%$ and chest X-ray is normal in $20-50 \%$ of cases. ${ }^{10,11}$ Although radiological tests such as brain Computed Tomography (CT) scan or Magnetic Resonance Imaging (MRI) are helpful, they may be normal in early stages and are not widely available in resource limited settings. Lumbar cerebrospinal fluid (CSF) is the most valuable test. The CSF proteins are elevated and may be markedly raised to $0.4 \mathrm{~g} / \mathrm{L}$ 
to $5.0 \mathrm{~g} / \mathrm{L}$ secondary to hydrocephalus and spinal block. However, ventricular CSF may record normal chemistry and cells if drawn from a site proximal to the inflammation and obstruction. Leukocytes in CSF are increased to $10-500$ cells $/ \mathrm{mm}^{3}$, with majority having lymphocyte predominance. However, neutrophils may predominate initially which can confuse the picture. The gold standard in diagnosis of TBM is positive culture of Mycobacteria tuberculosis from CSF which has both a long turn around time and a low smear and mycobacteria culture positive rate except if 10 to $20 \mathrm{ml}$ of CSF are supplied to the laboratory. ${ }^{12}$ Newer tests like the GeneXpert with a quick turn around time have been found to have a high sensitivity on sputum even in children. ${ }^{13}$ However, studies show a reduced sensitivity of GeneXpert in CSF compared to sputum. ${ }^{14}$

Other tests that would support TBM diagnosis like sputum or gastric aspirates also have low culture yields. ${ }^{5}$ To standardize diagnosis, a consensus case definition using clinical presentation, laboratory and radiological findings was made to guide clinicians and researchers. ${ }^{15}$ Outcome of TBM is generally poor with mortality among children ranging between 13-69\%. ${ }^{9,16,17}$ Younger age, tonic posturing, papilloedema, focal neurological deficit and stage at presentation were found to affect the prognosis. ${ }^{16,17}$

Red Cross War memorial Children's Hospital (RCWMCH) is a high TB and HIV burden setting with the prevalence of HIV and TB of $27.6 \%$ and $16.7 \%$ respectively among admitted patients on general paediatric wards. ${ }^{18}$ With this high prevalence, there was a need to document the diagnosis and treatment outcome of TBM in this hospital. We set out to describe our experiences in the presentation, diagnostic rigor, treatment and outcome of TBM at discharge from RCWMCH in the year 2009.

\section{Methodology}

This retrospective study was conducted at RCWMCH, a tertiary referral hospital affiliated to the University of Cape Town in South Africa. The study was approved by the Research ethics Committee of the University of Cape Town. The entry point was the hospital database which was used to generate a list of children who had been diagnosed with TBM from $1^{\text {st }}$ January 2009 to $31^{\text {st }}$ December 2009. The clinical notes of these patients were reviewed and relevant clinical data relating to the presentation, diagnosis, management and outcome of the children extracted using a pre-tested, pre-coded study tool.
We obtained the results of radiological examinations such as chest radiographs and brain CT scans or MRIs from the patient folders. Laboratory results were extracted from the National Health Laboratory Services electronic database. These included cerebrospinal fluid chemistry, microscopy, mycobacterial culture and antibiotic sensitivity, HIV test, sputum microscopy and mycobacterial culture results. The recently published consensus case definition of TBM was used to evaluate diagnostic certainty. ${ }^{15}$ This diagnostic criteria uses the clinical presentation and diagnostic tests to categorize TBM into Definitive, Probable, Possible and No TBM. Those who had a diagnosis of TBM in the folder but did not qualify according to the consensus case definition were excluded. Definite TBM was considered when Mycobacterium tuberculosis is isolated from CSF. Probable and Possible TBM were diagnosed according to consensus case definition for TBM for use in future clinical research. ${ }^{15}$ Probable TBM was considered when: 1) a patient presented with clinical features of meningitis and 2) suggestive CSF findings of TBM (total white cell count $>5$ cells $\times 10^{6} / \mathrm{L}$, protein $>0.45 \mathrm{~g} / \mathrm{L}$ and glucose $<2.2 \mathrm{mmol} / \mathrm{L}$ ), plus 3) one or more of the following i) chest radiograph findings consistent with pulmonary $T B$, ii) an extra-meningeal specimen positive for AFB, iii) other evidence of extra-meningeal TB (e.g. abdominal ultrasound features) or iv) brain computed tomography (CT) evidence of TBM including one or more of the following: basal meningeal enhancement, hydrocephalus or infarctions. Possible TBM was diagnosed when: 1) a patient presented with clinical features of meningitis and either 2) four or more of the following were present i) a history of TB ii) a predominance of CSF lymphocytes $(>50 \%)$, iii) illness duration of more than five days iv) CSF glucose $<2.2 \mathrm{mmol} / \mathrm{L}, \mathrm{v})$ altered consciousness, vi) clear or yellow CSF with protein $>1 \mathrm{~g} / \mathrm{L}$, vii) focal neurological signs, or 3) 'markedly abnormal' CSF (excluding isolated hypoglycemia) with evidence of TB elsewhere.

For children who were transferred to other hospitals in Cape Town to complete their treatment, those hospitals were visited and their treatment outcomes at discharge recorded. The outcome of treatment variables were improvement with / without neurological sequelae, or death. Poor outcome was defined as death or neurological sequelae at discharge. The neurological sequelae were obtained from the doctors' notes and it included physical neurological sequelae.

The Mantoux skin test is regarded as reactive as defined 
by guidelines of the World Health Organization: in high risk children (including HIV-infected children and severely malnourished children), $>5 \mathrm{~mm}$ of induration, and in all other children (whether they have received a BCG vaccination or not), $>10 \mathrm{~mm}$ of induration. ${ }^{19}$

TBM staging was undertaken using the modified criteria of the British Medical Research Council: ${ }^{20}$ in which TBM Stage I is defined as (Glasgow Coma Scale (GCS) 15 with no focal neurologic signs), TBM stage II is (GCS 11-14 or GCS of 15 with focal neurologic deficit) and TBM stage III is (GCS <11).

\section{Statistical Analysis}

Data was entered anonymously into an excel spreadsheet. To assess the accuracy of the data entered, a random sample of the original forms was compared with a computer print-out. Data was analyzed using STATA statistical package (StataCorp, Version 11). Descriptive analysis using medians with inter-quartile ranges $(\mathrm{IQR})$ are described for continuous variables that are not normally distributed while means and standard deviations (SD) are used for normally distributed data. For categorical variables, proportions are depicted as percentages of cases for which data is available. Data were tested for normality and the appropriate statistical test was used to test for strength of association.

\section{Results}

Of 22,943 children admitted to RCWMCH during the study period, we identified 40 children newly diagnosed with TBM; an incidence rate of 1.7 per 1000 admissions. The median age was 32 months (IQR 9-78.5) ranging from 6 weeks to 12 years. The male: female ratio was 1:1. Clinical features present at admission are summarized in table 1.

Table 1: Clinical features at presentation

\begin{tabular}{|c|c|c|c|}
\hline \multicolumn{2}{|l|}{ Presentation } & Frequency* & Percentage \\
\hline \multicolumn{2}{|l|}{ History of Fever } & $25 / 30$ & $83 \%$ \\
\hline \multicolumn{2}{|l|}{ Temperature above $37.5^{\circ} \mathrm{C}$} & $14 / 38$ & $37 \%$ \\
\hline \multicolumn{2}{|c|}{ Altered level of consciousness } & $23 / 32$ & $72 \%$ \\
\hline \multicolumn{2}{|l|}{ Convulsions } & $12 / 16$ & $75 \%$ \\
\hline \multicolumn{2}{|l|}{ Irritability } & $11 / 13$ & $85 \%$ \\
\hline \multicolumn{2}{|l|}{ Poor feeding } & $17 / 20$ & $85 \%$ \\
\hline \multicolumn{2}{|l|}{ TB contact } & $15 / 32$ & $47 \%$ \\
\hline \multicolumn{2}{|l|}{ Headache } & $14 / 15^{\#}$ & $93 \%$ \\
\hline \multicolumn{2}{|l|}{ Vomiting } & $20 / 28$ & $71 \%$ \\
\hline \multicolumn{2}{|l|}{ Cough } & $13 / 16$ & $81 \%$ \\
\hline \multicolumn{2}{|l|}{ Difficulty in breathing } & $7 / 26$ & $26 \%$ \\
\hline \multicolumn{2}{|l|}{ Failure to thrive } & $22 / 25$ & $88 \%$ \\
\hline \multicolumn{2}{|l|}{ Meningism } & $23 / 30$ & $77 \%$ \\
\hline \multicolumn{2}{|l|}{ Bulging fontanelle } & $4 / 8$ & $50 \%$ \\
\hline \multicolumn{2}{|l|}{ Neurological deficits } & $21 / 34$ & $62 \%$ \\
\hline Admission Fever Duration & 1-7 days & 14 & $74 \%$ \\
\hline$(\mathrm{N}=19)$ & $7-10$ days & 5 & $26 \%$ \\
\hline
\end{tabular}

*The denominator varies according to available data. ${ }^{\#}$ Only those above 4 years

Common features included fever, vomiting, meningism, neurological deficits, altered level of consciousness and failure to thrive. Although 25/30 (83\%) had a history of fever, $14 / 38(37 \%)$ had a temperature of $37.5^{\circ} \mathrm{C}$ and above, one had $34.5^{\circ} \mathrm{C}$ and $23 / 38(61 \%)$ had a normal temperature between 35 and $37.4^{\circ} \mathrm{C}$ at admission. Of the 32 with data on level of consciousness, only 18 had a record on the Glasgow coma scale. Of these 18, 9 had TBM stage 2 and 9 had TBM stage 3 according to the British Medical Research Council. ${ }^{20}$
According to the consensus case definition of $\mathrm{TBM}^{12}, 6$ (15\%) had definitive TBM, 17 (42\%), probable TBM and $18(44 \%)$ possible TBM. Thirty-five patients (87\%) were screened for HIV, of whom only $3(10 \%)$ were infected. No patients were receiving antiretroviral therapy (ART) at the time of TBM diagnosis. In addition, two were HIV exposed but their HIV DNA/PCR test was negative. Only 20/40 had available records on immunization and they all had received BCG vaccination.

Table 2 summarizes the CSF results. 
Table 2: Cerebrospinal Fluid Findings

\begin{tabular}{|c|c|c|}
\hline Results & Frequency* & Percentage \\
\hline CSF TB culture positive & $6 / 22$ & $27 \%$ \\
\hline AAFBs negative ${ }^{\#}$ & $22 / 22$ & $100 \%$ \\
\hline Raised proteins & $35 / 38$ & $92 \%$ \\
\hline $\begin{array}{l}>0.4 \mathrm{~g} / 1 \\
\text { Increased cell count }>5 \text { cells } / \mathrm{mm}^{3} \\
\text { Lymphocyte predominance } \\
\text { Neutrophil predominance }\end{array}$ & $\begin{array}{l}38 / 39 \\
32 / 39 \\
7 / 39\end{array}$ & $\begin{array}{l}97 \% \\
82 \% \\
18 \%\end{array}$ \\
\hline
\end{tabular}

The CSF protein concentration results were available in only 38 out of 40 children. Of these, $92 \%$ had raised protein concentration in CSF above $0.4 \mathrm{~g} / 1$ but $23(62 \%)$ had markedly raised proteins above $1.0 \mathrm{~g} / \mathrm{l}$. Ninety seven percent had above 5 cells $/ \mathrm{mm}^{3}$ as shown in table 2 below. Cells were predominantly lymphocytes in $33(83 \%)$. There were no results of blood glucose, but 33/38 (87\%) had CSF glucose below $3.0 \mathrm{mmol} / \mathrm{l}$. Other tests done to make a diagnosis of tuberculosis including Mantoux test, chest X-rays, sputum smear and culture, and CT and MRI are summarized in table 3 below.

Of the 30 who had data on chest X-ray findings, abnormal findings were present in $24(71 \%)$. Seventeen $(57 \%)$ had findings suggestive of TB which included $5(29 \%)$ with hilar or mediastinal lymphadenopathy, 4 (24\%) with miliary picture, $4(24 \%)$ with hilar adenopathy and reticulonodular infiltrates, $2(12 \%)$ with lobar infiltrates, $1(6 \%)$ with consolidation and perihilar infiltrates and 1 $(6 \%)$ with consolidation and fine nodular opacities like miliary. Other abnormal chest X-ray findings included 3 with isolated right upper lobe consolidation, 3 with peri-hilar and hilar infiltrates and pneumonia and 1 with bronco pneumonic picture.

Neuroimaging tests revealed that 33/36 (92\%) had an abnormality on CT or MRI as shown on table 3.

Table 3: Results of other TB investigations

\begin{tabular}{lll}
\hline Investigation & Frequency* & Percentage \\
\hline Mantoux test reactive & $16 / 28$ & $57 \%$ \\
Chest X-ray suggestive of TB. & $17 / 30$ & $57 \%$ \\
TB sputum culture positive. & $3 / 21$ & $14 \%$ \\
TB sputum smear negative & $21 / 21$ & $100 \%$ \\
CT MRI results & & \\
Basal meningeal enhancement & $18 / 36$ & $50 \%$ \\
Hydrocephalus & $16 / 36$ & $44 \%$ \\
Cerebral infarcts & $12 / 36$ & $33 \%$ \\
Cerebral oedema & $11 / 36$ & $31 \%$ \\
Normal & $4 / 36$ & $11 \%$ \\
\hline
\end{tabular}

*The denominator varies according to available data

The commonest finding was basal meningeal enhancement which was present in $50 \%$ but $25 / 33(76 \%)$ had multiple abnormalities; 12 had 2 abnormalities, 9 had 3 abnormalities and 4 had 4 abnormalities.

\section{Management and Outcome:}

All the children were treated with oral rifampicin, isoniazid, pyrazinamide and ethionamide which is the standard treatment in the country. ${ }^{21}$ They all received glucocorticosteroids; 36 (90\%) received Prednisolone and 4/40 (10\%) received Dexamethazone. Mannitol was administered to $6 / 40(15 \%)$. TB treatment was started on day 1 of admission in 25/37(67.5\%), day 2 in 5/37 (13.5\%) and day 3 in $7 / 37(19 \%)$. Three of the patients had no clear records about the date of initiation of TB treatment. Surgery was performed on 10/40 $(25 \%)$ - an extraventricular drain (EVD) alone was inserted in 3 children, 6 children received a ventriculoperitoneal (VP) shunt alone and 1 child had both EVD and VP shunt. Six $(15 \%)$ children with communicating hydrocephalus received serial lumbar punctures to reverse raised intracranial pressure. All 6 children had poor outcome.

Children with poor outcome of death and neurological sequelae had a statistically longer hospital stay with a median of 30 days, IQR 16-40 days while those with good outcome had a median of 5 days, IQR 2-17 days (Mann Whitney p 0.001). From RCWMCH, 2 went 
home, 2 died and 36 were referred to another hospital to complete their treatment. Of this latter group, 21 were admitted to the local TB hospital, Brooklyn Chest Hospital and 15 admitted to general hospitals in Cape
Town.

Table 4 summarizes the outcome at final hospital discharge.

Table 4: Treatment Outcome

\begin{tabular}{lll}
\hline Outcome & Frequency & Percentage \\
\hline Improved without neurological sequelae. & $15 / 36$ & $42 \%$ \\
Improved with neurological sequelae & $18 / 36$ & $50 \%$ \\
Died & $3 / 36$ & $8 \%$ \\
\hline
\end{tabular}

The outcome of 4 children could not be established because they were transferred to other hospitals too early and we did not find the data on outcome at discharge.

The trends noted with poor outcome were having surgery; (8 versus 1$)$, serial LPs, (6 versus none), acetazolamine use ( 7 versus none) and longer mean hospital stay. There was a strong association between poor outcome (death and neurological sequale) and TBM stage 3 on admission, Fischer's exact test $\mathrm{p}<0.01$. There was no association found between poor outcome and other presentations on admission. These included HIV status, CSF white cell count, age, CSF chemistry (protein and glucose), microscopy and culture.

The neurological sequelae experienced by the 18 children included hydrocephalus, (11), motor deficits (11), cranial nerve palsies (9), deafness (2), epilepsy (1), cortical blindness (1). Twelve of them suffered multiple sequelae.

\section{Discussion}

TBM is a severe disease whose initial presentation in children is like other common childhood illnesses resulting in late diagnosis. Although it is usually associated with poor outcome, our study found better treatment outcome and several lessons that can be drawn and applied.

We found a mean age of 48 months and only 11 were infants under 1 year. This is comparable to what several studies indicate that TBM mainly affects young children with the mean age ranging between 23 and 49 months. ${ }^{9-}$ ${ }^{11}$ All the 20 children with immunization records had BCG vaccine which is not surprising because although BCG is protective against TBM, studies show that those who get TBM after vaccination have a better treatment outcome. $^{22}$

RCWMCH has a high HIV prevalence of $27.6 \%$ on general paediatric wards but it was surprisingly low at $9.7 \%$ among those with TBM. Previous studies showed that HIV is a risk factor for central nervous system TB. ${ }^{3,5,7,8,23}$ However, Gijs et al also found a low HIV prevalence of $3.8 \%$ among children with TBM in Western cape province. ${ }^{9}$ This could be explained by the high burden of TB in the region such that children are infected regardless of their HIV status.

We found that TBM in these children presented mainly with non specific common childhood diseases but the data on duration was generally deficient. Of the 19 who had duration of fever, all were affected for less than 10 days. Cough was acute in 8/15 (53\%) of children. Several studies have similar findings of short duration of symptoms. ${ }^{5,8-10}$ More severe symptoms like convulsions and impaired level of consciousness were present in $12 / 16(75 \%) \%$ and $23 / 32(72 \%)$ respectively. These percentages seem high because many did not have records and it can be hypothesized that the ones without records most likely did not have the symptoms. Paradoxically, failure to thrive was present among 22/25 $(88 \%)$ despite the short duration of symptoms in the majority. This is similar to Gitz et al's study. ${ }^{9}$ We could not determine missed opportunities of early treatment because of the retrospective design. This has been described by other studies. ${ }^{5,9}$

Despite the difficulties of diagnosis of TBM, our study demonstrated that almost all had a diagnosis made by day 3 of admission. This is because of the high index of suspicion and also the hospital had access to quick laboratory and radiological tests which helped make a quick diagnosis and also start treatment early.

Definite TBM was present in only 15\% (Mycobacterium tuberculosis isolated from CSF). ${ }^{15} \mathrm{~TB}$ culture positive rates in CSF were lower in this study because only one sample was used. Daniel et al found that culture positive rates can go up from $53 \%$ on the first specimen to $83 \%$ with the third specimen. ${ }^{24}$

\section{Outcome of treatment}

Of the 36 children with available treatment outcome at discharge from hospital, 15/36, (42\%) improved 
without neurological sequelae, 18/36, (50\%) improved with neurological sequelae and 3/36, (8\%) died. Our results were different from Gitz et al in another retrospective study on the outcome of paediatric TBM after 6 months of treatment which was conducted between January 1985 and April 2005 in South Africa. In their study, the clinical outcome of the 554 patients was as follows: normal $(16 \%)$, mild sequelae $(52 \%)$, severe sequelae $(19 \%)$, and death (13\%). ${ }^{9}$ Unfortunately, we did not grade the neurological sequelae due to the deficient data in our retrospective study. Outcome would also be different at the end of treatment compared to the time of hospital discharge when the neurological outcome is still evolving. The mortality in our study was $8 \%$ which is much lower than has been described in other studies where mortality ranges between 13\% and 69\% even in developed countries. ${ }^{8-10,25-28}$ The better outcome in this study may be because all the 37 with records on date of starting antiTB treatment (ATT) had started treatment within 3 days of admission. Early initiation of treatment has been established as the main prognostic factor that predicts disease lethality and sequelae..$^{29-30}$ In addition, only 9 had TBM stage III basing on the GCS although 23 had impaired level of consciousness at admission. Stage III is associated with worse outcome., ${ }^{9,16,17}$ Availability of radiological tests like CT and MRI which were performed in 36/40 (90\%) and were abnormal in $89 \%$ of cases further strengthened the diagnosis and then early treatment. Since TB prevalence is high in this region, TBM is always held in high suspicion and treatment was started early with good results.

In addition, the prevalence of HIV was low in the study. HIV was not associated with poor outcome probably because the numbers were very few. HIV co-infection has been associated with poor treatment outcome of TBM in children. ${ }^{9}$

Like in other studies, we found that TBM stage III was associated with increased mortality., ${ }^{9}, 16,17$ Our study was not powered to look for associations but those who had surgery, serial LPs, acetazolamine use and longer mean hospital stay had poor outcome of death and neurological sequelae. Similarly, longer hospital stay has been associated with poor outcome. ${ }^{31}$ Misra et al also found that hydrocephalus and shunt surgery were poor prognostic features. ${ }^{17}$

The strength of this descriptive study is that we used a retrospective chart review to attain

data on a disease known to have very poor outcome and found better outcome than other studies. Our findings suggest that it is possible to reduce mortality and to improve outcome from TBM with rigorous diagnostics and early treatment initiation.

Limitations of our study included the retrospective design in which data recording was not standardized and as such some information was missing. In addition, we used the neurological sequelae recorded by the doctors in the folders which had missing data and could not therefore grade it. Another limitation was with our entry point which was the hospital records department. Some TBM diagnoses may have been missed out just as we found that some codes were in error.

\section{Conclusions}

We found that TBM mainly presented with acute non specific symptoms but the rigorous diagnostics helped make a quick diagnosis and start early treatment. Outcome of treatment at discharge was good with less than $10 \%$ mortality and half with neurological sequelae at discharge from hospital. Poor outcome was associated with TBM stage III disease.

\section{Recommendations}

We recommend a prospective study on the same topic so that the sequelae can be well documented. Since the presentation of TBM is non specific, clinicians in high TB endemic regions should have a high index of suspicion among all children with or without HIV infection. On suspicion of TBM, TB treatment should be started in the first 2 days of admission to improve the outcome.

\section{References}

1. World Health Organization. Global tuberculosis report 2012. Available at: www.who.int/tb/publications/ global_report/2012.

2. Harries AD, Dye C. Tuberculosis. Ann Trop Med Parasitol. 2006;100(5-6):415-31).

3. McShane H. Co-infection with HIV and TB: double trouble. Int J STD AIDS. 2005;16:95-101. doi: 10.1258/0956462053057576.PubMed [Cross Ref].

4. Baghaie N, Khalilzade S, Velayati AA et al. Extra pulmonary tuberculosis in children: two years study. Acta Med Iran. 2010;48(4):239-43).

5. Donald PR, Schoeman JF. Central nervous system tuberculosis in children. Chapter 38, 413-423 in textbook; Tuberculosis, a comprehensive clinical reference by $\mathrm{H}$ Simon Schaaf and Alimuddin I Zumla. 
6. Berman DS, Kibel M, Fourie PB. Childhood tuberculosis and Tuberculous meningitis: high incidence rates in the Western Cape of South Africa. Tuber Lung Dis 1992;6:311-406.

7. Donald PR, Cotton MF, Schaaf HS et al Pediatric Meningitis in the Western Cape Province of South Africa. Journal of Tropical Pediatrics. 1996 42(5):256-261.

8. GERMS-South Africa Surveillance Report, South Africa 2007. Bacterial and fungal meningitis amongst children < 5years, South Africa.

9. Gijs T. J. van Well, Berbe F. Paes, Johan F. Schoeman et al. Twenty Years of Pediatric Tuberculous Meningitis: A Retrospective Cohort Study in the Western Cape of South Africa Pediatrics Vol. 123 No. 2009, pp. e1-e8 (doi:10.1542/peds.2008-1353.

10. Farinha NJ, Razali KA, Holzel H, Morgan G, Novelli VM. Tuberculosis of the central nervous system in children: a 20-year survey. J Infect. 2000;41(1):61-68.

11. Yaramiçs A, Gurkan F, Elevli M, et al. Central nervous system tuberculosis in children: a review of 214 cases. Pediatrics. 1998; 102(5). Available at: www.pediatrics.org/cgi/content/full/102/5/e49.

12. Thwaites GE, Cahu TTH, Farrar JJ. Improving the bacterialogical diagnosis of Tuberculous meningitis. Int J Tuberc Lung Dis 2004 ;42: 378-379

13. Sekadde MP, Wobudeya E, Musoke P et al. Evaluation of the Xpert MTB/RIF test for the diagnosis of childhood pulmonary tuberculosis in Uganda: a crosssectional diagnostic study. BMC Infectious diseases 2013; 13:133 doi:10.1186/1471-2334-13-133

14. Taylor N, Gaur RL, Banaei N et al. Can a simple flotation method lower the limit of detection of Mycobacterium tuberculosis in extrapulmonary samples analyzed by the GeneXpert MTB/RIF assay? J Clin Microbiol. 2012 Jul;50(7):2272-6. doi: 10.1128/JCM.01012-12. Epub 2012 May 2.

15. Marais S, Thwaites G, Marais JB et al. Tuberculous meningitis: a uniform case definition for use in clinical research. Lancet Infec Dis 2010 (10): 803-812

16. Mahadevan B, Mahadevan S, Tiroumourougane Serane V. Prognostic Factors in Childhood Tuberculous Meningitis. Journal of Tropical Pediatrics 2002; 48(6):362365.

17. Misra UK, Kalita J, Mandal SK et al. Prognosis of tuberculous meningitis: a multivariate analysis. Journal of the Neurological Sciences Volume 137, Issue 1, April 1996,
Pages 57-61

18. Weakley M, Vries A, Eley B. S et al. HIV infection, tuberculosis and workload in a general Paediatric Ward. SAJCH 2009, 3:2.

19. World Health Organization. Guidance for national tuberculosis programmes on the management of tuberculosis in children. 2006.

20. British Medical Research Council. Streptomycin treatment of tuberculous meningitis. Br Med J. 1948;1 :582-597

21. Department of Health. South Africa. National Tuberculosis Guidelines. 2009.

22. Kumar P, Kumar R, Srivastava KL, Kumar M. Protective role of BCG vaccination against tuberculous meningitis in Indian children: a reappraisal. Natl Med J India. 2005 Jan-Feb;18(1):7-11.

23. Rana F S, Hawken M P, Lucas S B.et al. Autopsy study of HIV-1-positive and HIV-1-negative adult medical patients in Nairobi, Kenya. J Acquir Immune Defic Syndr. 2000;24:23-29.

24. Daniel TM New approaches to the rapid diagnosis of tuberculous meningitis. I Infect Dis1987; 155:599_ 603.

25.Anne-Sophie Christensen H, Åse Andersen B, Isik Johansen $\mathrm{S}$ et al. Tuberculous meningitis in Denmark: a review of 50 cases BMC InfectiousDiseases 2011, 11:47.

26. Padayatchi N, Bamber S, Bobat R et al. Multidrugresistant tuberculous meningitis in children in Durban, South Africa. Pediatr Infect Dis J. 2006; (25): 147-50.

27. Porkert MT, Sotir M, Blumberg HM et al. Tuberculous meningitis at a large inner-city medical center. $A m$ J Med Sci 1997, 313: 325-31.

28. Paganini H, Gonzalez F, Santander C, Casimir L, Berberian G, Rosanova MT. Tuberculous meningitis in children: clinical features and outcome in 40 cases. Scand J Infect Dis. 2000;32(1): 41-45.

29. Farinha NJ, Razali KA, Holzel H, Morgan G, Novelli VM. Tuberculosis of the central nervous system in children: a 20-year survey. J Infect 2000;41:61-68.

30. Nunes C, Cunha S, Gomes I, Lucena R, Moraes D, Melo A. Prognostic factors of tuberculous meningoencephalitis lethality. Arq Neuropsiquiatr 1998;56:772-777.

31. Gimenes Rodrigues M, Lin J, Soares T, Minett C et al. Prognostic factors predicting a fatal outcome in HIV-negative children with neurotuberculosis. Arq. Neuro-Psiquiatr. 2010; 68 (5): 755-760. 